\title{
Long-term influence of chitin concentration on the resistance of cement pastes determined by atomic force microscopy
}

Article in Physica Status Solidi (A) Applications and Materials · July 2016

DOI: $10.1002 /$ pssa.201600105

CITATIONS

0

8 authors, including:

José Antonio Sánchez

Tecnológico de Monterrey

12 PUBLICATIONS 13 CITATIONS

SEE PROFILE

Jorge Luis Menchaca

Autonomous University of Nuevo León

25 PUBLICATIONS 98 CITATIONS

SEE PROFILE
READS
Miguel Ángel Gracia Pinilla

Autonomous University of Nuevo León

47 PUBLICATIONS 264 CITATIONS

SEE PROFILE 


\title{
Long-term influence of chitin concentration on the resistance of cement pastes determined by atomic force microscopy
}

\author{
Eduardo Ortega', Oliver Rodríguez-Martínez', Miguel Figueroa-Labastida', Andrés Alberto Villa-Pulido', \\ Antonio Sánchez-Fernández², Rodrigo Cué-Sampedro', Miguel Angel Gracia-Pinilla ${ }^{3,4}$, \\ and Jorge Luis Menchaca*,

\footnotetext{
${ }^{1}$ Department of Physics, Tecnológico de Monterrey, Av. Eugenio Garza Sada 2501, 64849 Monterrey, Nuevo León, Mexico

${ }^{2}$ Department of Chemistry, Tecnológico de Monterrey, Av. Eugenio Garza Sada 2501, 64849 Monterrey, Nuevo León, Mexico

${ }^{3}$ Faculty of Physical and Mathematical Sciences, Universidad Autónoma de Nuevo León, Av. Universidad s/n, 66451, San Nicolás de los Garza, Nuevo León, Mexico

${ }^{4}$ Centro de Investigación en Innovación y Desarrollo en Ingeniería y Tecnología, Universidad Autónoma de Nuevo León, Km 10, Nueva
} \\ carretera Internacional de Monterrey, 66600 Apodaca, Nuevo León, Mexico
}

Received 19 February 2016, revised 20 May 2016, accepted 27 June 2016

Published online 25 July 2016

Keywords atomic force microscopy, cement, chitin, elastic properties, nanoindentation

*Corresponding author: e-mail jorge.menchacarr@uanl.edu.mx, Phone: +52 8183294030, Fax: +52 8183522954

The presence of sulfates potentialize damage on cementbased materials, leading to structural failures. Therefore, structures must be designed to compensate for this effect. The mechanical properties of cement-chitin mixtures are investigated with different percentages of chitin $(0.5,1.3$, and $2.1 \mathrm{wt} . \%$ ) and aging of composite in a joint nanoscopic- and macroscopic-scale by experimental study. The objective is to increase the durability of concrete elements at coastal aquifers where concrete structures are in constant exposure to sulfate ions, chloride ions among others. Tapping mode AFM was used to characterize the surface structure and roughness of the cement pastes. To verify the chitin addition and the formation of sulfate-based aggregates Raman and IR spectra were recorded and are presented in this work. Then, force spectroscopy was used to obtain the nanomechanical properties at three different exposure times ( 1 day, 6 months, and 1 year) into water or a $\mathrm{SO}_{4}{ }^{-2}$ environment. Macroscopic parameters (e.g., compression strength of cylindrical probes) were assessed for comparison following standard guidelines. The results show a decrease of its mechanical properties as a function of the polymer concentration but more importantly, they correlate the elasticity and adhesion at the nanoscale with the behavior of the bulk material.

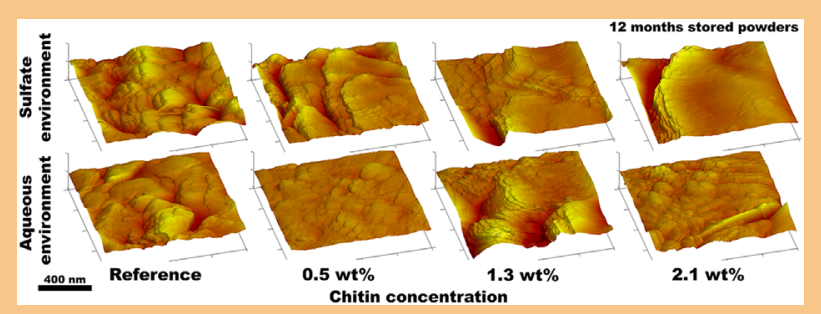

1 Introduction Cement is a mixture used in the construction field, of different substances that might change the cure, shrinkage, and mechanical properties of the final product [1]. One of the most important ingredients of cement is the hydrated calcium silicate phase $(\mathrm{C}-\mathrm{H}-\mathrm{S})$ that is responsible for its main physical and chemical properties [2]. During its production and use, cement releases greenhouse gas emissions both directly (heating of limestone) and indirectly (heating the kiln). As a result, research is being done on new sustainable concrete mixtures that can improve not only its mechanical properties, but also reduce its environmental footprint. A popular approach to attain this is by adding a very low amount of multiwalled carbon nanotubes (MWCNT) or carbon nanofibers (CNFs), 
at concentrations of $0.025-0.08 \mathrm{wt} \%$ of cement, which has been demonstrated to increase the strength and stiffness of cement up to $30 \%$ [3]. Also, in order to achieve a nonvibration behavior of a building, steel fibers can be added to counteract early age cracking of concrete that has a positive effect due to the restrained drying shrinkage [4].

As different additives were tried, it has become more important to implement experimental techniques to test the behavior or response of the composite materials. In that respect, for assessing direct and reliable nanoscale data, the AFM allows measurement of interaction forces between different phases, and quantification of different physical properties like force, adhesion, or friction [2]. Two different paths can be followed to calculate the mechanical properties of the cement.

The first has to do with a phase change between the material's distinguishable areas. Velez et al. sensed the mechanical properties of Portland cement by the resonance frequency technique [5]. A comparison between the macroscopic-scale results and the microscopic results made the consistent finding that porosity is inversely proportional to the modulus of elasticity.

The second relies in the tip-sample contact forces. The adhesion-force measurements obtained in this way are dependent upon the accuracy of experimental measurements for the probe stiffness, probe-tip radius, pull-off deflection, and cut-off distance [6]. Knowing these values, the Young's modulus of the sample can be calculated using a Hertz model, it approximates the sample as an isotropic and linear elastic solid. It considers the contact between two spherical bodies, and can be used when the materials do not influence each other [7]. Jones et al. published their findings about the stiffness of cementitious materials and $\mathrm{C}-\mathrm{H}-\mathrm{S}$ using the AFM, based on a comparison between the numeral analytical modeling and the experimental indentation procedure [8].

While the methodology to acquire the experimental data is critical, it is also necessary to consider the working environment. Recent studies include different environments to analyze how these affect the cementitious materials, especially the effect by marine systems on coastal regions. The temperature, $\mathrm{pH}$ solution, time, and duration of the exposure are important in the rate of expansion of the C-H-S [9]. Ranjani and Ramamurthy tested the sulfate attacks on the foam concrete, observing a deterioration on the cementing material exposed to magnesium sulfates higher than the one exposed to sodium sulfates. Němeček et al. investigated the alkali activation of slag, metakaolin, fly ash, and aluminosilicate materials, by using the elastic unloading part of the experimental load-penetration curves $[10,11]$.

With the aim of improving the durability of concrete elements exposed to chemically aggressive environments, in this article a cement-chitin mixture is analyzed. Chitin is the main component of the cell walls of fungi, the exoskeletons of crustaceans and insects, and internal shells of cephalopods. The compound is a linear polysaccharide consisting of residues of $\beta-1,4-\mathrm{N}$-acetyl-D-glucosamine and it is classified into three crystalline forms whose applications are found elsewhere [12]. The purpose of this work is to interpret the qualitative data on the elasticity modulus and roughness of cement powders with different chitin concentrations and aging. The nanostructure technique is appropriate to characterize the new generation of a hybrid of nanoscale $\mathrm{C}-\mathrm{S}-\mathrm{H}$ that can increase the durability of concrete [13].

2 Experimental After cellulose, chitin and its derivatives are the most abundant structural polysaccharides. This biopolymer have been used as an additive for many biocements. The materials investigated gained benefit in terms of (i) enhanced injectability; (ii) increased setting time; (iii) augmented thickening effect; and (iv) retention of heavy metals [14-16]. By changing the dosing and setting time of the cement-composite material an assortment of its mechanical properties is attained.

The experimental procedure consists of measuring the interaction forces acting between a probe and the sample with an atomic force microscope (AFM) in air. A judicious choice of the nature of probe and substrate is necessary, the probe and substrate should not react to ensure the stability and reproducibility of force measurements. Moreover, the surface of the substrate must be flat to avoid roughness effects.

2.1 Instrumentation Analyses were done using a commercial AFM (NanoScope IIIa Controller, Veeco Metrology Group, Santa Barbara, CA) along with its MultiMode SPM 4.31ce software. All force values were calculated using nonconductive silicon nitride $\left(\mathrm{Si}_{3} \mathrm{~N}_{4}\right)$ triangular cantilevers (NP-20 Veeco Co., Santa Barbara, CA) with a length of $120 / 205 \mu \mathrm{m}$ and nominal spring constants of $0.24-0.06 \mathrm{~N} \mathrm{~m}^{-1}$. The roughness analysis was carried out by comparing the images obtained in Tapping Mode $^{\mathrm{TM}}$ using nonconductive silicon rectangular cantilevers (MPP-11100 Veeco Co.) with a length of $125 \mu \mathrm{m}$ and a nominal frequency of $300 \mathrm{kHz}$. AFM images were obtained under ambient conditions; height, amplitude, and phase images were recorded simultaneously. Images were recorded with typical scan speeds of 1 line $\mathrm{s}^{-1}$ using a scan head with a maximum range of $148 \times 148 \mu \mathrm{m}^{2}$.

Scanning electron micrographs of the samples were obtained in low vacuum with a Nova NanoSEM200 (FEI, Hillsboro, Oregon) using a low-vacuum (LV) detector. For determining the bulk properties of the systems, analyzed compressive strength were carried out with a Tester Press (S.A.E. Ibertest, Madrid, Spain).

Infrared spectra were acquired with a IRAffinity-1 spectrophotometer instrument (Shimadzu Corp., Kyoto, Japan), spectroscopy measurements were performed on an ATR module (ATR - miracle single-reflection horizontal accessory) in the spectral region of $500-4000 \mathrm{~cm}^{-1}$ using a spectral resolution of $4 \mathrm{~cm}^{-1}$ and a scan average of 45 . Data were collected using IRSolutions version 1.6 (Shimadzu Software). 
The employed Raman instrument was a Horiba Jobin Yvon iHR320 (Horiba Ltd., Kyoto, Japan) spectrometer, adapted with an optical microscope (Olympus Corp., Shinjuku, Japan) for adjusting the working distance. Spectra were recorded on the cement-chitin powders with a green $535-\mathrm{nm}$ laser and an 1800 lines $\mathrm{mm}^{-1}$ grating working with a spectral resolution of $9 \mathrm{~mm}^{-1}$. The laser beam with a total power of $75 \mathrm{~mW}$ was focused onto the sample with a $100-\mu \mathrm{m}$ focal spot that serves to avoid any damage to the material and to obtain a more representative area to overcome the inherent heterogeneity of the samples. Spectra were analyzed by using the profile baseline removal of the program Spectrovis and recorded in the frequency ranges $100-1200 \mathrm{~cm}^{-1}$ and $3000-4000 \mathrm{~cm}^{-1}$ in order to investigate the four Raman-active vibration modes of sulfate anions, the " $\mathrm{Al}(\mathrm{OH}) 6$ " octahedron vibration and the hydrogen-bond network due to the water and hydroxyl stretching representative of ettringite and gypsum.

All experiments were performed in air, the temperature of the surrounding wall was maintained at $24^{\circ} \mathrm{C}$ in a humidity-controlled atmosphere: midhumidity $(30 \%)$. Measurement of the relative humidity $(\mathrm{RH})$ was achieved with a precision of $1 \%$ and a range of 25-95\% (Traceable 4184 Jumbo Humidity/Temperature Meter; Control Co., Friendswood, TX).

2.2 Sample preparation In this work, commercial $\beta-1,4-\mathrm{N}$-acetyl-D-glucosamine, generally known as chitin (Sigma-Aldrich part C9213) was introduced into cement powders in a proportion of $0.5,1.3$, and $2.1 \%$ (by weight of cement), these samples were stored in closed plastic containers with water or a solution of sulfate ions at $20^{\circ} \mathrm{C}$ to study the sulfate-resisting Portland cements exposed to marine environments. Their physical properties were measured with an AFM at three different exposure times (1 day, 6 months, and 1 year) in order to measure a difference in their performance.

Pastes from these powders were prepared at a demineralized water-to-cement ratio (w/c) of 0.50. After mixing, the cement pastes were cast into cylindrical-shaped molds and compacted by vibration. The specimens were demolded after 2 days at $90-92 \%$ relative humidity. The samples were directly stored in a sodium sulfate solution of $0.5 \mathrm{M}$ (several sets of 40 samples per $1.7 \mathrm{~L}$ of the sodium sulfate solution) at $20^{\circ} \mathrm{C}$ and held there for as long as one month.

The macromechanical properties of our mixture samples were assessed by compressive strength measurements, at 1 and 28 days. Some guidelines followed for the performed measurements of this cement composite material are marked in the ASTM standard C39, which is the case of the specimens dimensions ( 100 by $200 \mathrm{~mm}$ ) and the load application (platen to crosshead measurement) corresponding to a stress rate of $0.25 \pm 0.05 \mathrm{MPa} \mathrm{s}^{-1}\left(35 \pm 7 \mathrm{psi} \mathrm{s}^{-1}\right)[17]$.

2.3 Force measurements The interaction forces were directly measured between the tips and cement powders. The results gathered from the piezoelectric sensor in the AFM can be interpreted in a force-distance curve by monitoring the vertical displacement of the cantilever $\left(\delta_{\mathrm{c}}\right.$ or deflection) with respect to the piezovoltage applied $\left(Z_{\mathrm{p}}\right)$ [18]. Then, the tip-sample force can be expressed in terms of Hooke's law

$$
F=-k_{\mathrm{c}} \delta_{\mathrm{c}},
$$

in which for characterizing the spring constant of the cantilever $\left(k_{\mathrm{c}}\right)$ the thermal tune method (that relates thermal noise to the mean square deflection) was used [19].

Elasticity measurements were obtained from force plots acquired (120 per zone) at different points of the samples. Then, relating the experimental graphs obtained with the standard deflection of the cantilever on a rigid substrate (calibration curve) the tensile modulus in each plot was obtained using a customized MATLAB program with a modified Hertz model that correlate the data of the applied force $F$ and the indentation depth $\delta$ of the tip into the samples:

$$
\delta_{\mathrm{s}}^{3}=\left(\frac{3}{4 E_{\mathrm{TOT}}}\right)^{2} \frac{F^{2}}{R}
$$

where $R$ can be approximated as the indenter radius for small indentations [20]. It can be noted that an effective elastic modulus of the system $\left(E_{\mathrm{TOT}}\right)$ is obtained. Following Johnson insights [21], knowing the probe values one can obtain the sample Young's modulus:

$$
\frac{1}{E_{\mathrm{TOT}}}=\frac{1-v_{\mathrm{t}}^{2}}{E_{\mathrm{t}}}+\frac{1-v_{\mathrm{s}}^{2}}{E_{\mathrm{s}}},
$$

where the subindices refer to tip and sample values. Values of Young's modulus, Poisson ratio, and tip radius for $\mathrm{Si}_{3} \mathrm{~N}_{4}$ cantilevers were $290 \mathrm{GPa}, 0.3$ and $20 \mathrm{~nm}$, respectively [22].

3 Results and discussion When referring to "sulfate attack," a detriment of the material involving any type of sulfate interactions with cement paste is denoted, independently of the curing temperature and sulfate source. The experiments does not include damage, which is not specially recognized to the chemical sulfate attack (e.g., crystallization of water-soluble sulfate salts) [23].

3.1 Imaging of cement composites The purpose of AFM and SEM imaging in this work is to find a change in the conglomerates and aggregation of cement phases, particularly the identification of ettringite and gypsum as they are the primary products of the chemical reaction between a sulfate-bearing solution and cement hydration products [24]. Ettringite formation is considered to be the cause of most of the expansion and disruption of concrete structures involved in the sulfate attack $[25,26]$. When ettringite forms later (even years), the expansion in a very rigid hardened concrete can produce cracking, alteration of paste composition, and spalling. An external sulfate attack 
typically occurs when dissolved sulfate penetrates the concrete (e.g., seawater, oxidation of sulfide minerals in clay, or bacterial action).

AFM surface plots were acquired and the numerical data obtained from the experiments are provided in subsequent sections. Some fiber-like structures were observed during scanning the samples, these structures can be appreciated in the scanning electron microscopy (SEM) image of Fig. 1C, and were related to ettringite formation by subsequent studies by Raman spectroscopy.

3.2 Roughness analysis Roughness takes a leading role in determining how the material will interact with its environment. Roughness is often a good predictor of the performance of a material, as irregularities in the surface may form nucleation sites for cracks or corrosion. Alternatively, roughness may enhance adhesion.

Roughness measurements of different aging time and chitin dosing acquired with the AFM working in tapping mode are available in the Supporting Information (online at: www.pss-a.com), a summary of these values are listed in Table 1. Chitin shows a tendency to reduce the surface texture. However, the reduction of surface roughness over time suggests aging as the dominant factor.

These facts may be correlated with the dosagedependent performance of this polymer: a thickening action, proved by the slump reduction [27], and the formation of agglomerates in the particle size distribution. In the presence of large amounts of biopolymer, steric repulsions between the chains of the chitin could take place, resulting in its plasticizing action and in the increase in the surrounding free water, which entailed a water-retention ability reduction [28].

3.3 Infrared and Raman spectra Infrared spectroscopy is a fast and reliable technique to detect not only crystalline but also the amorphous phases that can develop on certain cementitious materials. The infrared radiation is absorbed by molecules and transformed into a mechanical vibration; when the incident energy equals the energy of a particular molecular vibration, absorption occurs. Figure 2 shows the FTIR spectra of cement-chitin composites with

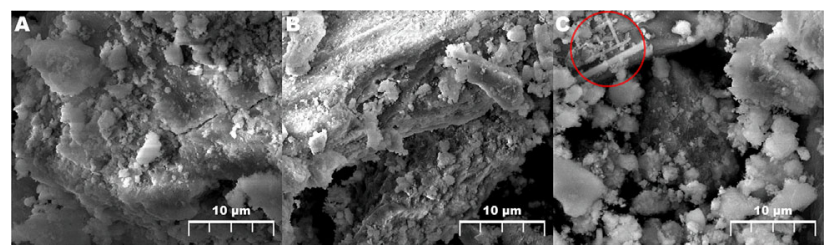

Figure 1 Scanning electron microscopy images of cement powders. (A) Reference sample, exposed during 1 year in an aqueous environment. (B) $2.1 \mathrm{wt} \%$ of chitin, exposed during 1 year in an aqueous environment. (C) $2.1 \mathrm{wt} . \%$ of chitin, exposed during 1 year in a sulfate environment, fiber-like structures, related to a hydrous calcium aluminum sulfate mineral generally known as ettringite, are highlighted.
Table 1 Quadratic mean roughness (in $\mathrm{nm}$ ) of cement powders with different concentrations of chitin and aging times on a sulfate environment.

\begin{tabular}{lllll}
\hline conditions & REF & 0.5 wt. $\%$ & 1.3 wt.\% & 2.1 wt. $\%$ \\
\hline 1 day & $139 \pm 11$ & $88 \pm 11$ & $32 \pm 5$ & $106 \pm 20$ \\
6 months & $79 \pm 12$ & $44 \pm 4$ & $65 \pm 11$ & $64 \pm 12$ \\
12 months & $58 \pm 6$ & $34 \pm 8$ & $43 \pm 9$ & $59 \pm 13$ \\
\hline
\end{tabular}

different compositions and aging times. Vibration frequencies characteristic of aluminum hydroxide (Al-OH) in the form of gibbsite and a $v_{4} \mathrm{SO}_{4}$ mode from gypsum were located at 970 and $668 \mathrm{~cm}^{-1}$, respectively [29]. The wide band observed from 1480 to $1420 \mathrm{~cm}^{-1}$ is generated by the $\mathrm{CO}_{3} \quad v_{3}$ vibrations associated with the presence of calcium monocarboaluminate $\left(\mathrm{C}_{4} \mathrm{AC}_{\mathrm{C}} \mathrm{H}_{11}\right)$ in the composite. The anhydrous $\mathrm{C}_{4} \mathrm{~A}_{3} \check{\mathrm{S}}$ spectrum also showed the presence of sulfates, with a band located at $1100 \mathrm{~cm}^{-1}$ related to the $v_{3}$ $\mathrm{SO}_{4}$ vibration zone.

As ettringite $\left(\mathrm{C}_{6} \mathrm{~A} \stackrel{\text { S }}{H_{32}}\right)$ structure is based on columns of cations of the composition $\left(\mathrm{Ca}_{3}\left[\mathrm{Al}(\mathrm{OH})_{6}\right] \cdot 12 \mathrm{H}_{2} \mathrm{O}\right)^{3+}$ it presents a very strong antisymmetrical stretching frequency of the sulfate ion $\left(v_{3} \mathrm{SO}_{4}\right)$ centered toward $1120 \mathrm{~cm}^{-1}$, which overlaps with the anhydrous $\mathrm{C}_{4} \mathrm{~A}_{3} \check{\mathrm{S}}$ band. Another frequency associated with this phase is the presence of aluminate bands (Fig. 2: REF $12 \mathrm{M}$ vs. $2.1 \%$ 6M) near 855 and $550 \mathrm{~cm}^{-1}$ (not shown) as an indication of Al-O-H bending and Al-O stretching, respectively [30].

The $\mathrm{C}=\mathrm{O}$ stretching region of the ester groups (between 1790 and $1740 \mathrm{~cm}^{-1}$ ) are indicators of the incorporation of $\mathrm{N}$-acetyl glucosamine and suggest the formation of the

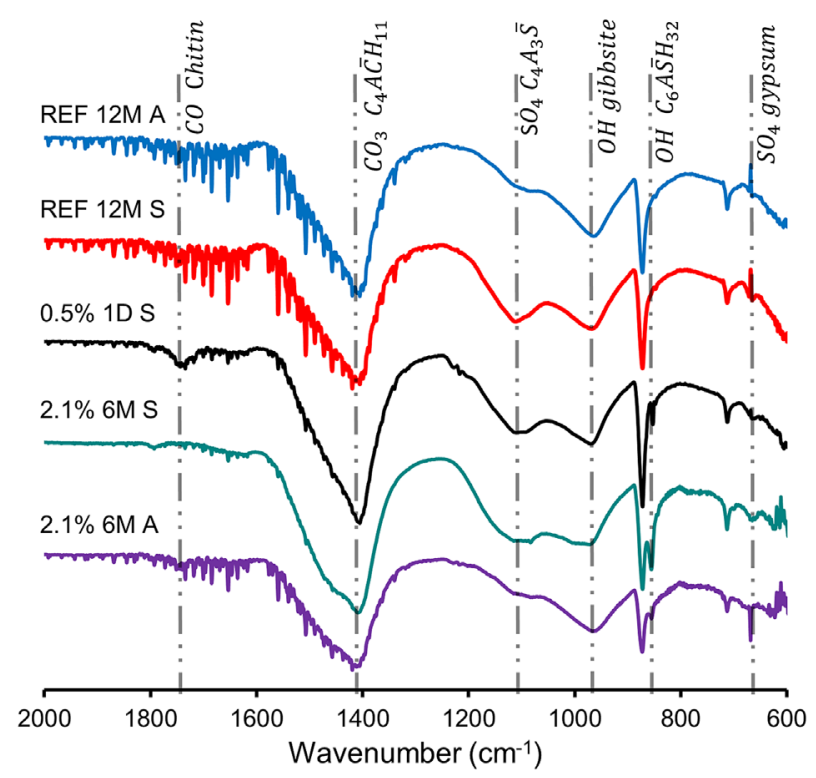

Figure 2 FTIR spectra recorded in the range $600-2000 \mathrm{~cm}^{-1}$ for selected cement-chitin powders, obtained at different concentrations and aging times. 
composite [31, 32]. From the set of samples displayed in Fig. 2, the absorption band is only clearly visible in sample $0.5 \%$ as the noise level in that region of the spectra is too high.

Raman spectroscopy is a useful tool to detect ettringite development. For sulfated cement hydrates, Raman spectroscopy has been used for describe the hydrogenbonding network and local environment of sulfate anions [25]. Figure 3 presents the Raman spectra acquired for the cement composite samples. This type of spectra possess four active vibration modes of sulfate $(988,414,1120$, and $620 \mathrm{~cm}^{-1}$ ), which corresponds to the vibrational peaks of gypsum [33]. The signal recorded in the longest range is used to investigate the $\mathrm{O}-\mathrm{H}$ elongation from hydroxyl anions and water molecules (structural bonded or structural free).

For the samples investigated, during the first days, chitin incorporation is visible due to the intensity of the $3630 \mathrm{~cm}^{-1}$ peak. Moreover, the gypsum phase is visible as it already exists in the raw materials. In the cement-chitin system, the ettringite formation appears to be greater than in the reference samples as suggested not only for the strong presence of sulfates in the system but also the lack of the $\mathrm{OH}$-stretching peak and the broader water spectral peak found in the reference $\left(3300-3470 \mathrm{~cm}^{-1}\right)$. Regarding the chitin dosing, the spectra obtained do not provide much information about the samples qualitative phase distributions, as there is no difference in the spectra obtained at different concentrations (even after repeated testing on different samples) as the noise level due fluorescence is on the way. In the $3300-3700 \mathrm{~cm}^{-1}$ region, there is slight evidence of the diminution of $\mathrm{OH}$ bonding, which could

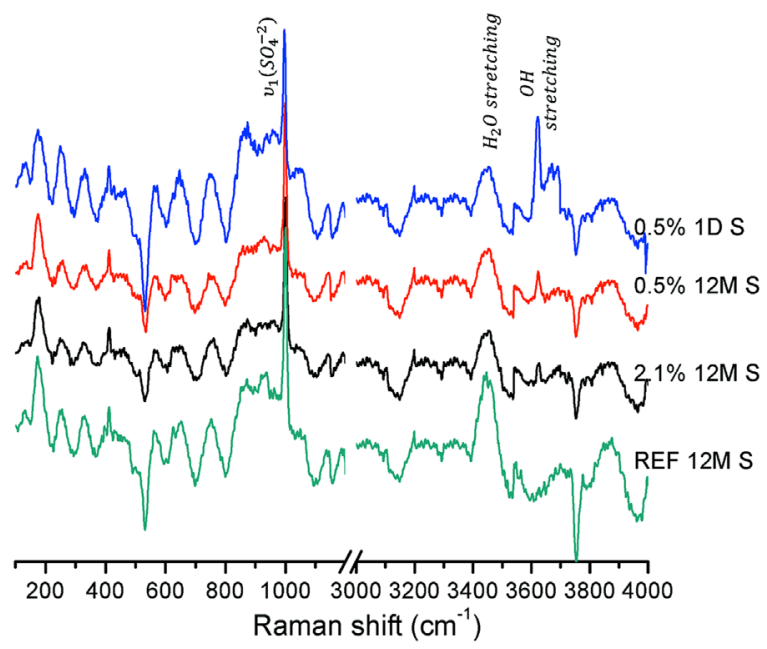

Figure 3 Raman spectra recorded in the range $100-4000 \mathrm{~cm}^{-1}$ for selected cement-chitin powders. One Raman-active vibration mode of sulfate anions $\left(988 \mathrm{~cm}^{-1}\right)$, and the hydrogen bond network due to the water $\left(3300-3470 \mathrm{~cm}^{-1}\right)$ and hydroxyl $\left(3630 \mathrm{~cm}^{-1}\right)$ stretching representative of ettringite and gypsum are labeled. reveal some information about the ongoing hydration process formation of delayed sulfoaluminate phases [34].

3.4 Elasticity and adhesion analysis To achieve sufficient sensitivity in force measures, cantilevers with low spring constant values have been implemented. The force-separation curves presented in this article are limited to the range of $10-60 \mathrm{~nm}$ to increase the resolution of the measurements, however, in each case, the piezoelectric ramp size is approximately $1 \mu \mathrm{m}$. In these experiments, the tip and the surface are retained in contact for $1 \mathrm{~s}$. The full force applied by the probe reaches $70-80 \mathrm{nN}$. In the elastic region, the approaching (or extending) deflection curve was used to achieve further calculations owing to its lack of adhesion (Fig. 4).

A hysteresis appears between the extending and retracting curves as a result of the adhesion of the tip onto the sample where positive force values are related to repulsion. The adhesion force is measured at the lowest point of the plot before the tip separates from the substrate. The interaction distance (related to the attraction force present) between the $\mathrm{Si}_{3} \mathrm{~N}_{4}$ tip and the cement-polymer surface diminish as a function of the polymer addition, which will be later related to the diminution of the elasticity of the composite (for individual samples records please refer to the supplementary material).

Figure 5 shows the elasticity and adhesion for the sulfate systems. The measurement error is assessed from the standard deviation during the acquisition of at least 360 data points. The addition of chitin slightly reduces this attraction (in about $5 \mathrm{fJ}$ ), revealing a repulsion within the region of interaction between the tip and the surface. It is observed that aging is a determinant factor as values present a shorter range of interaction force that can be related to the stabilization and equilibria of steric and/or electrostatic forces [35]. In addition, $\mathrm{OH}$ and $\mathrm{NH}_{2}$ groups would undergo ionization under alkaline conditions, becoming negatively charged. As a consequence, its charge density should be

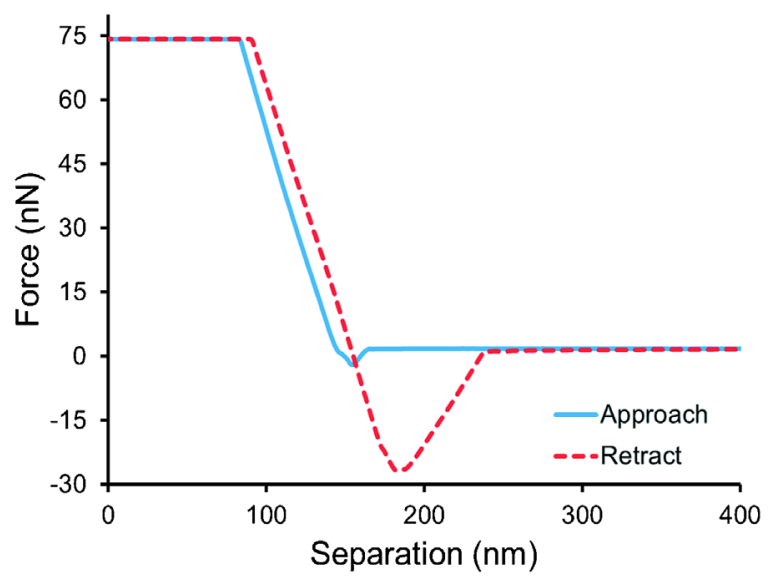

Figure 4 Experimental force-separation curve from a cementchitin powder showing attractive interaction and deflection of the cantilever due to surface interaction. 


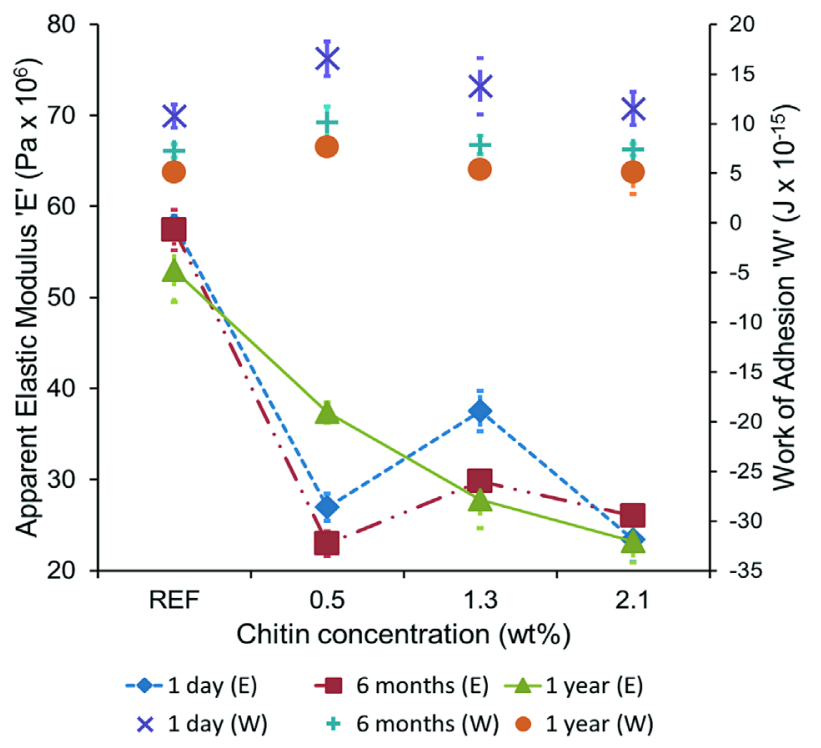

Figure 5 Elasticity $E$ and work of adhesion $W$ plot of cement powders with various concentrations of chitin kept at different exposure times in a sulfate environment.

larger and responsible for the appearance of electrostatic repulsions between chains of the polymer, which inhibit intermolecular association resulting in a lower attractive force into the AFM tip. These results are in agreement with the reports by Lasheras-Zubiate et al. [36], where chitosan addition led to a charge inversion of the zeta potential (high positive values) of the composite, suggesting a formation of agglomerates of cement particles and polymer molecules negatively charged, sheltered by a layer of positive counterions (calcium ions).

As the geometry of the tip does not allow a precise calculation of the contact area interaction, the corresponding disposition of the chitin chains or cement aggregates in the powder is not predictable. From this perspective, the AFM force plots here denote a qualitative idea of the interactions that take place in these systems, and cannot be employed as quantitative results. However, since a diminution in the repulsion force was detected as chitin was added, even at low dose, one can assume that in these conditions an electrostatic repulsion takes part [37]. It could be hypothesized that the repulsion in this situation is due to a superplasticizer effect of chitin chains interposing between the compression between the tip and the powder in a subsuperficial region, as loosely (not bound) components would be easily removed by the tip oscillations while gathering the data.

With regard to the elasticity modulus, the introduction of chitin strongly affects the elasticity of cement. Recently, several authors have indicated the relevant role of the hydrogen bridge bonding in the mechanism of the surface modification of concrete [38-40], in our case, we suggest that the large hydroxyl groups of chitin can modify the mechanical properties of the cementitious materials. In a water or sulfate environment, this can be appreciated
Table 2 Compression resistance (in MPa) of cement cylinders with different concentrations of chitin and aging times on a sulfate environment.

\begin{tabular}{llll}
\hline conditions (days) & REF & 0.5 wt.\% & 1.3 wt.\% \\
\hline 1 & 15.8 & 14.1 & 12.8 \\
28 & 35.1 & 34.2 & 30.7 \\
\hline
\end{tabular}

physically in the samples' morphology (Table 1) where the RMS roughness of the cements powders with chitin present lower values than the control samples (cements without chitin); we find the nonlinearity of the Young's modulus in function of the chitin concentration.

When elasticity values are compared with the macroscopic compression test (Table 2) chitin's tendency to decrease compression resistance as a function of the polymer added is also present. Published reports indicate that cement pastes with chitosan show high viscosity at low shear rates but exhibit a reduction when great stress is applied [41]. The fall in viscosity is explained because linkages between polymer and cement particles may break, and failure propagation can align with the linear chains direction. When this behavior is extrapolated with the compression test, chitin introduction, which facilitates retention of water into the composite and therefore [36], in an atmosphere rich of sulfonates, generates anionic exchange (as observed in the decreased carbonates IR signal when compared with reference samples), can later converge into the promotion of microcracks and the formation of weaker chitin-based domains.

Although cylinder fracture experiments show a drop of less than 25\%, nanocompression tests results diminish almost by half regardless aging time. This suggests that ettringite plays a marginal role in the interaction of agglomerates with cement phases making the biopolymer addition the main component that affects the rheology of cement. One hypothesis is that chitin's hydrogen bonds increase the water demand of powders, which affects the hydration process. Moreover, chitin might have the tendency to attach at the aggregates surfaces, thus decreasing the surface roughness and hence resulting in a lower shear between two adjacent large grains later on. Such effect has been observed on chitosan-cement admixture and has been related to both a set-retarding ability and water retention [36].

4 Conclusions In this work, by fitting a Hertz model into the approach-force curve, the Young's modulus of a cement-powder composite was obtained. The long-term evolution of these samples was assessed using two spectrometry techniques IR and Raman to demonstrate chitin addition and the later ettringite presence. We found that there is a systematic decrease of the elasticity for cement-chitin samples regardless of the confinement environment. The work of adhesion was evaluated with 
the area between the retraction curve and the reference line for all the samples. An aging effect was found for the work of adhesion showing a diminution as a function of time.

In summary, we reported a useful statistical nanoindentation method to measure the elastic properties of a cement-powder composite with chitin in agreement with the standard procedures (compressive test) at the macroscale. Further study should be focused in the impact of the tip material; especially in the case of ferrous tips to reflect a more realistic macroscopic verification, since steel reinforces concrete structures as in the case of structural beams, headframes, and pillars.

Supporting Information Additional supporting information may be found in the online version of this article at the publisher's website.

Acknowledgements The authors thank MSc. Daniel Cruz Lemus and Dr. Elías Perez for their help with the IR and Raman acquisition. Also we thank Consejo Nacional de Ciencia y Tecnología (CONACYT) for the project \#169319 and CAT-120, CAT-128 Research Programs by Tecnológico de Monterrey for the support at this research.

\section{References}

[1] P. Mondal, S. P. Shah, and L. Marks, Cem. Concr. Res. 37, 1440-1444 (2007).

[2] C. Plassard, E. Lesniewska, I. Pochard, and A. Nonat, Langmuir 21, 7263-7270 (2005).

[3] Z. S. Metaxa, J. W. T. Seo, M. S. Kontsa-Gdoutos, M. C. Hersam, and S. P. Shah, Cem. Concr. Comp. 34, 612-617 (2012).

[4] V. Corinaldesi and G. Moriconi, Cem. Concr. Comp. 33, 596-601 (2011).

[5] K. Velez, S. Maximilien, D. Damidot, G. Fantozzi, and F. Sorrentino, Cem. Concr. Res. 31, 555-561 (2001).

[6] G. Lomboy, S. Sundararajan, K. Wang, and S. Subramaniam, Cem. Concr. Res. 41, 1157-1166 (2011).

[7] Determining the elastic modulus of biological samples using the atomic force microscope, Application Note for JPK Instruments AG, Berlin, Germany, 2008.

[8] C. A. Jones, Z. C. Grasley, and J. A. Ohlhausen, Cem. Concr. Comp. 34, 468-477 (2012).

[9] G. I. S. Ranjani and K. Ramamurthy, Cem. Concr. Comp. 34, 825-834 (2012).

[10] J. Němeček, V. Similauer, and L. Kopecky, Cem. Concr. Comp. 33, 163-170 (2011).

[11] W. C. Oliver and G. M. Pharr, J. Mater. Res. 7, 1564-1583 (1992).

[12] C. P. Souza, B. C. Almeida, R. R. Colwell, and I. N. G. Rivera, Mar. Biotechnol. 13, 823-830 (2011).

[13] P. J. M. Monteiro, A. P. Kirchheim, S. Chae, P. Fischer, A. A. MacDowell, E. Schiable, and H. R. Wenk, Cem. Concr. Comp. 31, 577-584 (2009).

[14] Z. H. Pan, H. P. Cai, P. P. Jiang, and Q. Y. Fan, J. Polym. Res. 13, 323 (2006).
[15] C. Muzzarelli and R. A. A. Muzzarelli, J. Inorg. Biochem. 92, 89 (2002).

[16] M. H. lkhraisat, C. Rueda, L. Blanco, F. T. Mariño, J. Torres, U. Gbureck, and E. Lopez, Acta Biomater. 6, 257 (2010).

[17] ASTM Standard C39/C39M - 12a, Standard Test Method for Compressive Strength of Cylindrical Concrete Specimens (ASTM International, West Conshohocken, PA, 2003), DOI: 10.1520/C0039_C0039M-12A.

[18] G. Haugstad and W. L Glaedfelter, Ultramicroscopy 54, 31-40 (1994).

[19] J. L. Hutter and J. Bechhoefer, Rev. Sci. Instrum. 64, 1868-1873 (1993).

[20] A. Fischer-Cripps, Introduction to Contact Mechanics, 2nd edn. (Springer, Berlin, 2000), pp. 103-108.

[21] K. L. Johnson, Contact Mechanics (Cambridge University Press, UK, 1985), pp. 91-93.

[22] A. Khan and J. Philip, J. Appl. Phys. 95, 1667-1672 (2004).

[23] H. Haynes, R. O’Neil, and P. K. Mehta, Concr. Int. 18, 63-68 (1996).

[24] P. K. Mehta, Sulfate Attack on Concrete - A Critical Review, in: Material Science of Concrete III (American Ceramic Society, Westerville, OH, 1992), pp. 105-130.

[25] G. Renaudin, Y. Filinchuk, J. Neubauer, and F. GoetzNeunhoeffer, Cem. Concr. Res. 40, 370-375 (2010).

[26] M. Collepardi, Concr. Int. 21, 69-74 (1999).

[27] L. Patural, P. Marchal, A. Govin, P. Grosseau, B. Ruot, and O. Devès, Cem. Concr. Res. 41, 46-55 (2011).

[28] P. Yan, X. Qin, W. Yang, and J. Peng, Cem. Concr. Res. 31, 1285-1290 (2001).

[29] L. Fernández-Carrasco, D. Torrens-Martín, L. M. Morales, and S. Martínez-Ramírez, Infrared Spectroscopy - Materials Science, Engineering and Technology (InTech, Croatia, 2012), ISBN: 978-953-51-0537-4.

[30] M. J. Sánchez-Herrero, A. Fernández-Jiménez, and A. Palomo, Cem. Concr. Res. 46, 41-49 (2013).

[31] J. Brugnerotto, J. Lizardi, F. M. Goycoolea, W. ArguÈellesMonal, J. DesbrieÁres, and M. Rinaudo, Polymer 42, 3569-3580 (2001).

[32] K. Prabu and E. Natarajan, Adv. Appl. Sci. Res 3, 1870-1875 (2012).

[33] S. Martinez-Ramirez, M. Frías, and C. Domingo, J. Raman Spectrosc. 37, 555-561 (2006).

[34] N. Garg, K. Wang, and S. W. Martin, Cem. Concr. Res. 53, 91-103 (2013).

[35] L. Ferrari, J. Kaufmann, F. Winnefeld, and J. Plank, J. Colloid Interface Sci. 419, 17-24 (2014).

[36] M. Lasheras-Zubiate, I. Navarro-Blasco, J. M. Fernández, and J. I. Alvarez, J. Appl. Polym. Sci. 120, 242-252 (2011).

[37] L. Ferrari, J. Kaufmann, F. Winnefeld, and J. J. Plank, J. Colloid Interface Sci. 347, 15-24 (2013).

[38] A. Stewart, B. Schlosser, and E. P. Douglas, ACS Appl. Mater. Interfaces 5, 1218-1225 (2013).

[39] S. Choi, S. Maul, A. Stewart, H. R. Hamilton, and E. P. Douglas, Polym. Eng. Sci. 53, 283-294 (2013).

[40] A. Hashim, Functional Polymers: Nanocomposite Materials from Microfibrillated Cellulose, in: Advances in Nanocomposite Technology (Intech, Croatia, 2011), chap. 14, pp. 319-333.

[41] A. Yahia and K. H. Khayat, Cem. Concr. Res. 31, 731 (2001). 